\title{
Experiencia en el tratamiento de la hemorragia uterina anormal en adolescentes con enfermedad renal crónica
}

\author{
Juana Serret-Montoya ${ }^{1}$, Miguel A. Villasís-Keever², Alejandra Aguilar-Kitsu³, Iliana Del Moral-Estrada ${ }^{3}$, \\ Abigail Hernandez-Cabezza ${ }^{4}$ y Jessie N. Zurita-Cruz ${ }^{2,5 *}$ \\ ${ }^{1}$ Servicio de Medicina del Adolescente, Hospital Infantil de México Federico Gómez; ${ }^{2}$ Unidad de Investigación en Epidemiología Clínica, Unidad \\ Médica de Alta Especialidad (UMAE) Hospital de Pediatría, Centro Médico Nacional Siglo XXI, Instituto Mexicano del Seguro Social; ${ }^{3}$ Departamento \\ de Nefrología Pediátrica, Unidad Médica de Alta Especialidad (UMAE) Hospital de Pediatría, Centro Médico Nacional Siglo XXI, Instituto Mexicano \\ del Seguro Social; ${ }^{4}$ Clinica de Ginecología Pediátrica, Unidad Médica de Alta Especialidad (UMAE) Hospital de Pediatría, Centro Médico Nacional \\ Siglo XXI, Instituto Mexicano del Seguro Social; ${ }^{5}$ Unidad de Investigación en Nutrición, Unidad Médica de Alta Especialidad (UMAE) Hospital de \\ Pediatría, Centro Médico Nacional Siglo XXI, Instituto Mexicano del Seguro Social. Ciudad de México, México
}

\begin{abstract}
Resumen
Introducción: La enfermedad renal crónica (ERC) se asocia con alteraciones menstruales, y el manejo del sangrado uterino suele ser complejo por las condiciones de este grupo de pacientes. El objetivo de este trabajo fue describir la respuesta clínica al tratamiento hormonal de las alteraciones menstruales de adolescentes con ERC. Métodos: Se presentan los datos de una serie de casos de pacientes adolescentes con ERC que cursaron con alteraciones menstruales y que recibieron tratamiento desde el año 2008 al 2012. Se identificaron las características del trastorno menstrual, del tratamiento hormonal recibido y de la respuesta al mismo. El análisis estadístico fue descriptivo. Resultados: Se estudiaron 11 pacientes de sexo femenino con edad promedio de 14.5 años, que se encontraban en prediálisis $(n=1)$, diálisis peritoneal $(n=7)$ y hemodiálisis $(n=3)$. Las pacientes presentaron hiperpolimenorrea asociada a la opsomenorrea $(n=3)$, en su mayoría clasificadas como hemorragia uterina anormal secundaria. El tratamiento, en general, fue con progestágenos de manera inicial (clormadinona con o sin medroxiprogesterona) o bien con anticonceptivos combinados. En la mayoría de las pacientes se obtuvo una respuesta favorable; sin embargo, hubo casos en los que fue necesario modificar la dosis y el tiempo de tratamiento. Conclusiones: La mayor parte de las adolescentes con ERC que han sido tratadas por hemorragia uterina anormal en nuestro estudio tuvieron una respuesta favorable al tratamiento hormonal.
\end{abstract}

Palabras clave: Hemorragia uterina. Falla renal. Adolescentes.

\section{Experience in the treatment of uterine hemorrhage in adolescents with chronic renal disease}

\section{Abstract}

Background: Chronic kidney disease (CKD) is associated with menstrual abnormalities and management of uterine bleeding is often complex because of the conditions in this group of patients. The aim of this study was to describe the clinical response to hormonal treatment of menstrual alterations in adolescents with CKD. Methods: We present data of cases of 
adolescent patients with CKD who had undergone menstrual changes and received treatment during the period 2008 to 2012. The characteristics of the menstrual disorder, hormone treatment received, and response to treatment were evaluated. The statistical analysis aplicated to analyze the results was descriptive. Results: We studied 11 patients with a mean age of 14.5 years, who were in predialysis $(n=1)$, peritoneal dialysis $(n=7)$, hemodialysis $(n=3)$. Patients had hyperpolymenorrhea associated with opsomenorrhea $(n=3)$, mostly classified as secondary abnormal uterine bleeding. Treatment, in general, was with progestins initially (chlormadinone with or without medroxyprogesterone) or combined contraceptives. In the majority of the patients, a favorable response was obtained; however, there were cases where it was necessary to modify the dose and time of treatment. Conclusions: The majority of adolescents with CKD who have been treated for abnormal uterine bleeding in our study had a favorable response to hormonal treatment.

Key words: Uterine hemorrhage. Renal failure. Adolescents.

\section{Introducción}

Las mujeres con enfermedad renal crónica (ERC) sufren una variedad de problemas ginecológicos que con frecuencia no son reconocidos ${ }^{1}$. Desde hace varios años se ha establecido que la ERC se asocia con trastornos neuroendocrinos, desaceleración del crecimiento y disfunción sexual, que pueden aparecer desde la pubertad ${ }^{1,2}$. En particular, sobre los problemas menstruales en pacientes con ERC, la progresión a la falla renal puede condicionar el deterioro del ciclo menstrual normal y conducir a ciclos irregulares y anovulatorios $^{3}$; el patrón menstrual típico en las mujeres con ERC es la oligomenorrea y la amenorrea, que puede ocurrir desde la menarca ${ }^{4}$.

A pesar del conocimiento que se tiene de los trastornos menstruales en las mujeres con ERC, en la actualidad aún no están del todo definidos los posibles mecanismos involucrados; los más estudiados son los aspectos endocrinológicos. Se ha documentado la ausencia de una adecuada liberación cíclica de la hormona liberadora de gonadotropina $(\mathrm{GnRH})$ por el hipotálamo, lo cual conduce a la pérdida del pulso normal de hormona luteinizante (LH), que resulta en una disminución de los niveles de estradiol y testosterona ${ }^{2,5,6}$. También se ha observado inhibición central en la liberación de gonadotropinas, con disminución de la secreción de estrógenos por el ovario. A medida que la enfermedad renal progresa, se instala un hipogonadismo hipogonadotrófico y amenorrea, con mayor riesgo de osteoporosis ${ }^{3}$. Por otro lado, la hiperprolactinemia es común en pacientes con ERC, ya que se ha descrito que ocurre del 20 al $80 \%$ de las pacientes. Los niveles de prolactina se elevan por aumento de su producción y disminución de su aclaramiento, lo que deriva en la supresión de las gonadotropinas y conduce a anovulación, ciclos menstruales irregulares e hipoestrogenemia y galactorrea ${ }^{7,8}$. Asimismo, la elevación de leptina se ha señalado como otro factor que contribuye a las anormalidades del ciclo menstrual, ya que influye en la liberación de $\mathrm{GnRH} \mathrm{H}^{9,10}$.

En general, el manejo del sangrado menstrual abundante en las adolescentes con ERC sigue las pautas que se han establecido para las pacientes sin enfermedad subyacente, donde la monitorización de la cantidad del sangrado a través del calendario menstrual coadyuva para establecer la gravedad del sangrado y proporcionar un tratamiento específico ${ }^{11,12}$.

Además, para el tratamiento de las alteraciones menstruales se debe considerar la etiología y el objetivo final del tratamiento. En este contexto, el objetivo del tratamiento hormonal en el manejo de las alteraciones menstruales es la inhibición del sangrado en un periodo de tiempo de 72 horas desde su instauración - la regularización del mismo durante los 3 meses después de su instauración. De esta forma, la piedra angular son los estrógenos y progestágenos, los cuales causan proliferación endometrial, aumentan el fibrinógeno y los factores de coagulación (V, VIII, IX), además de promover la agregación plaquetaria, con lo que se induce la hemostasia ${ }^{13,14}$.

En general, el tratamiento del sangrado menstrual anormal en adolescentes con ERC se basa en las guías descritas anteriormente, en vista de que no hay información o recomendaciones específicas para este grupo particular de pacientes. Es posible que la ausencia de recomendaciones se deba a que no hay estudios donde se haya descrito la efectividad de un tratamiento en particular en estos casos.

Después de realizar una amplia revisión de la literatura, se encontró que el estudio de las alteraciones menstruales en pacientes con ERC es muy limitado ${ }^{15-18}$, sobre todo con respecto al tratamiento. Desde hace varios años, en el Hospital de Pediatría del Centro Médico Nacional Siglo XXI se han identificado y tratado adolescentes con ERC que cursan con trastornos menstruales; sin embargo, no se dispone de la información global para determinar el impacto que ha 
tenido el tipo de tratamiento empleado. Por esta razón, el objetivo de este estudio fue describir la experiencia de la Clínica de Ginecología Pediátrica sobre la respuesta clínica al tratamiento hormonal de las adolescentes con ERC con hemorragia uterina anormal, evaluándola en las diferentes etapas de la enfermedad: prediálisis, diálisis peritoneal, hemodiálisis y trasplante renal.

\section{Métodos}

Se realizó un estudio de serie de casos de adolescentes con ERC estadio IV y V, de acuerdo con la escala de KDIGO (Kidney Disease: Improving Global Outcomes) ${ }^{18,19}$, atendidas entre 2008 y 2015. Se incluyeron pacientes adolescentes en prediálisis, diálisis peritoneal, hemodiálisis y trasplante renal, con sangrado o hemorragia uterina anormal y que recibieron tratamiento en la Clínica de Ginecología Pediátrica. Se excluyeron las pacientes con expedientes clínicos incompletos.

Después de una búsqueda intencionada en los diferentes registros de la clínica de Ginecología, se identificaron 15 adolescentes con ERC que fueron atendidas por sangrado uterino anormal; del total, solamente se pudieron recuperar 11 expedientes clínicos completos.

De los expedientes de las adolescentes que cumplieron con los criterios de selección, se registró la edad, peso, talla, tiempo de evolución y etiología de la ERC, e inicio y tipo de terapia de sustitución de las pacientes. También la edad de inicio del desarrollo puberal, estadio puberal en el momento de la evaluación y las características de los ciclos menstruales. De acuerdo con las notas médicas, se registró la evolución del sangrado menstrual, en el cual se especifica la frecuencia, cantidad y duración del ciclo menstrual o, de forma indirecta, en relación con el número de toallas sanitarias utilizadas por día. Esto se realizó con la finalidad de evaluar la respuesta clínica en cada una de las pacientes, sin perder de vista los objetivos del tratamiento, ya fuera con fines de inhibición (cese) completa de la hiperpolimenorrea dentro de las primeras 72 horas desde el inicio del sangrado, o bien la regularización del sangrado menstrual, con ciclos con una periodicidad de $28 \pm 7$ días, con una duración de sangrado menstrual de $4 \pm 2$ días y con una cantidad de sangrado de $60 \pm 20 \mathrm{ml}$ o la utilización de $<6$ toallas sanitarias $^{20}$. Asimismo, se identificó el tipo de tratamiento hormonal utilizado (estrógenos, progestágenos o combinados) y las dosis y ciclos recibidos con los cuales se regularizó el ciclo menstrual, con lo cual se definió la respuesta al manejo hormonal utilizado.

Antes del inicio del estudio, el proyecto se sometió a evaluación por el Comité Local de Investigación en Salud y se determinó su aprobación mediante el número de registro R-2012-3603-31.

\section{Medición de niveles de hormona estimulante de tiroides, hormona luteinizante, hormona folículo estimulante, estradiol y prolactina}

La LH, la hormona folículo estimulante (FSH), el estradiol, la prolactina, la hormona estimulante de la tiroides (TSH), triyodotironina y tiroxina se midieron por inmunoensayo electroquimioluminiscente (ECLIA). Todos los experimentos de ECLIA se determinaron usando COBAS 6000 e601 (Roche Diagnostics GmbH, Indianápolis, IN, EE.UU.) en duplicado, de acuerdo con las recomendaciones del fabricante. Los coeficientes de variación intra e interensayo para todas las mediciones fueron menores al $7 \%$. También se incluyó una curva estándar dentro de cada ensayo.

\section{Diagnóstico según el perfil hormonal}

El hipotiroidismo subclínico se definió por la elevación de la TSH sérica (entre 6 y $9.9 \mathrm{mu} / \mathrm{ml}$ ) y las hormonas tiroideas normales ${ }^{21}$; el hipotiroidismo se definió como la presencia de hormonas tiroideas normales 0 bajas y TSH > $10 \mathrm{mu} / \mathrm{ml}$; la disfunción ovárica, cuando la relación LH/FSH fue 2:1 o más; y la hiperprolactinemia, cuando los niveles de prolactina fueron mayores a $25 \mathrm{ng} / \mathrm{ml}^{22}$.

\section{Definición de alteraciones menstruales}

El sangrado menstrual se definió como polimenorrea cuando se presentó en un intervalo mayor a 21 días; como hipermenorrea, cuando el sangrado menstrual duró más de 7 días o fue excesivo (cantidad $>80 \mathrm{cc} 0$ $>6$ toallas por día); y como opsomenorrea o irregularidad menstrual, cuando el sangrado ocurrió en intervalos mayores a 34 días.

\section{Definición de falla al tratamiento}

Se definió falla al tratamiento en los siguientes casos: en las pacientes con sangrado abundante y persistencia del sangrado menstrual, después de 72 horas; en las pacientes con ciclos menstruales irregulares 
Tabla 1. Características generales de las 11 adolescentes con enfermedad renal crónica con trastornos menstruales

\begin{tabular}{|c|c|c|c|c|c|}
\hline Caso & $\begin{array}{l}\text { Edad en el momento de } \\
\text { la evaluación }\end{array}$ & Terapia de sustitución & $\begin{array}{c}\text { Años de evolución de la } \\
\text { ERC }\end{array}$ & $\begin{array}{l}\text { Estadio puberal } \\
\text { mamario }\end{array}$ & $\begin{array}{l}\text { Edad menarca } \\
\text { (años) }\end{array}$ \\
\hline $1^{*}$ & 13 a $11 \mathrm{~m}$ & $\mathrm{HD}$ & 1 & III & 11 \\
\hline $1^{*}$ & 15 a $11 \mathrm{~m}$ & DP & 3 & V & 11 \\
\hline 2 & 12 a $9 \mathrm{~m}$ & DP & 4 & IV & 11 \\
\hline 3 & 14 a & DP & 1 & IV & 12 \\
\hline 4 & 13 a $9 \mathrm{~m}$ & Sin terapia & 1 & IV & 12 \\
\hline 5 & $15 \mathrm{a}$ & DP & 4 & III & 15 \\
\hline 6 & $15 \mathrm{a}$ & DP & 7 & III & 12 \\
\hline 7 & 12 a $11 \mathrm{~m}$ & $\mathrm{HD}$ & 1 & IV & 13 \\
\hline 8 & $14 \mathrm{a}$ & $\mathrm{DP}$ & 7 & IV & 12 \\
\hline 9 & 12 a $7 \mathrm{~m}$ & DP & 1 & IV & 12 \\
\hline 10 & 14 a $11 \mathrm{~m}$ & $\mathrm{HD}$ & 2 & IV & 12 \\
\hline 11 & $16 \mathrm{a}$ & DP & 2 & IV & 12 \\
\hline
\end{tabular}

*Esta paciente tuvo dos evaluaciones en tiempos diferentes.

a: años; DP: diálisis peritoneal; ERC: enfermedad renal crónica; HD: hemodiálisis; m: meses.

que después de 3 meses de tratamiento persistan con sangrado menstrual con un intervalo menor a 21 días o mayor a 35 días, o con una duración mayor a 7 días, o en cantidad mayor a 6 toallas por día.

\section{Análisis estadístico}

Se realizó un análisis estadístico de tipo descriptivo; las variables se expresan como frecuencias simples y porcentajes. Se utilizó el programa SPSS v.17.0.

\section{Resultados}

De las 11 pacientes incluidas, la edad en el momento de la primera consulta por el problema menstrual fue variable, entre los 12 y los 16 años. De acuerdo con el momento de la primera evaluación, una paciente se encontraba en prediálisis, nueve en terapia de sustitución, siete en diálisis peritoneal (DP) y tres en hemodiálisis (HD). El tiempo de evolución de las ERC varió de uno a siete años. De acuerdo con el desarrollo puberal, las pacientes se clasificaron en Tanner mamario IV $(n=8)$ y Tanner III $(n=3)$ (Tabla 1).

En cuanto al motivo de la consulta, en las 11 pacientes se debió a sangrado menstrual abundante, considerado como hipermenorrea, hiperpolimenorrea o polimenorrea, y en todas las pacientes se determinó como causa del trastorno menstrual la hemorragia uterina anormal. En cuanto al tratamiento, en la mayoría de los casos inicialmente se trató de inhibir el sangrado mediante clormadinona o medroxiprogesterona $y$, posteriormente, con anticonceptivos orales combinados. El manejo de la hemorragia aguda con clormadinona consistió en dosis de $6 \mathrm{mg} /$ día durante tres días, $4 \mathrm{mg} /$ día durante otros tres días y $2 \mathrm{mg}$ cada 24 horas (Tabla 2).

Con el propósito de brindar una mejor perspectiva de la evolución de cada paciente y debido a lo heterogéneo de la presentación individual, a continuación se describe cada una de las pacientes.

\section{Pacientes con antecedentes de ciclos menstruales regulares}

Las pacientes con antecedentes menstruales regulares a los 2 años de edad ginecológica presentaron hemorragia uterina anormal. Particularmente, las pacientes 2 y 8 tuvieron una respuesta favorable con el uso de progestágenos; sin embargo, el resto de las pacientes presentaron falla al tratamiento inicial, por lo que se utilizaron otras opciones.

Caso 3. La paciente fue valorada por hiperpolimenorrea. Resultados de los exámenes de laboratorio: hemoglobina $(\mathrm{Hb}), 6 \mathrm{~g} / \mathrm{dl}$; ultrasonido pélvico, normal. En un inicio recibió manejo para la hemorragia aguda con clormadinona, con respuesta parcial; sin embargo, a la 
Tabla 2. Características generales de las 11 adolescentes con enfermedad renal crónica con trastornos menstruales

\begin{tabular}{|c|c|c|c|c|c|c|}
\hline Caso & $\begin{array}{l}\text { Motivo de } \\
\text { consulta }\end{array}$ & Perfil hormonal & $\begin{array}{l}\text { Diagnóstico } \\
\text { nosológico }\end{array}$ & Tipo de tratamiento & $\begin{array}{l}\text { Meses de } \\
\text { tratamiento }\end{array}$ & Evolución \\
\hline $1^{*}$ & Hipermenorrea & $\begin{array}{l}\text { Hiperprolactineamia } \\
\text { LH/FSH 10:1 }\end{array}$ & $\begin{array}{l}\text { HUA } \\
\text { Disfunción ovárica }\end{array}$ & Clormadinona & 4 & Respuesta favorable \\
\hline $1^{*}$ & Hiperpolimenorrea & Normal & HUA & $\begin{array}{l}\text { Medroxiprogesterona } \\
\text { AOC }\end{array}$ & $\begin{array}{l}1 \\
6\end{array}$ & Respuesta favorable \\
\hline 2 & Hiperpolimenorrea & LH/FSH 5:1 & $\begin{array}{l}\text { HUA } \\
\text { Disfunción ovárica } \\
\text { Hipotiroidismo } \\
\text { subclínico }\end{array}$ & $\begin{array}{l}\text { Clormadinona } \\
\text { Levotiroxina }\end{array}$ & $\begin{array}{c}4 \\
12\end{array}$ & Respuesta favorable \\
\hline 3 & Hiperpolimenorrea & Normal & HUA & $\begin{array}{l}\text { Clormadinona } \\
\text { Medroxiprogesterona }\end{array}$ & $\begin{array}{l}1 \\
7\end{array}$ & $\begin{array}{l}\text { Falla inicial, } \\
\text { posteriormente } \\
\text { respuesta favorable }\end{array}$ \\
\hline 4 & $\begin{array}{l}\text { Opsomenorrea } \\
\text { Hiperpolimenorrea } \\
\text { Dismenorrea }\end{array}$ & LH/FSH 8:1 & $\begin{array}{l}\text { HUA } \\
\text { Disfunción ovárica }\end{array}$ & Clormadinona & 4 & Respuesta favorable \\
\hline 5 & $\begin{array}{l}\text { Opsomenorrea } \\
\text { Hiperpolimenorrea }\end{array}$ & Normal & HUA & $\begin{array}{l}\text { AOC } \\
\text { Medroxiprogesterona }\end{array}$ & $\begin{array}{l}1 \\
3\end{array}$ & $\begin{array}{l}\text { Falla inicial por falta } \\
\text { de apego al } \\
\text { tratamiento, } \\
\text { posteriormente } \\
\text { respuesta favorable }\end{array}$ \\
\hline 6 & Polimenorrea & Normal & $\begin{array}{l}\text { HUA secundaria a } \\
\text { cistoadenoma } \\
\text { derecho }\end{array}$ & $\begin{array}{l}\text { Clormadinona } \\
\text { AOC }\end{array}$ & $\begin{array}{l}9 \\
3\end{array}$ & $\begin{array}{l}\text { Falla inicial, } \\
\text { posteriormente } \\
\text { respuesta favorable }\end{array}$ \\
\hline 7 & Hiperpolimenorrea & Normal & HUA & Clormadinona & 3 & Respuesta favorable \\
\hline 8 & Hiperpolimenorrea & Normal & HUA & $\begin{array}{l}\text { Clormadinona } \\
\text { Medroxiprogesterona }\end{array}$ & $\begin{array}{l}1 \\
8\end{array}$ & Respuesta favorable \\
\hline 9 & Hiperpolimenorrea & Normal & HUA & $\begin{array}{l}\text { AOC } \\
\text { Clormadinona } \\
\text { Medroxiprogesterona } \\
\text { Leuprolide }\end{array}$ & $\begin{array}{c}4 \\
1 \\
6 \\
12\end{array}$ & $\begin{array}{l}\text { Respuesta favorable } \\
\text { inicial, } \\
\text { posteriormente } \\
\text { difícil control }\end{array}$ \\
\hline 10 & Hiperpolimenorrea & LH/FSH 6:1 & $\begin{array}{l}\text { HUA secundaria a } \\
\text { disfunción ovárica }\end{array}$ & $\begin{array}{l}\text { Medroxiprogesterona } \\
\text { Clormadinona } \\
\text { AOC }\end{array}$ & $\begin{array}{l}1 \\
1 \\
2\end{array}$ & $\begin{array}{l}\text { Falla inicial, } \\
\text { posteriormente } \\
\text { respuesta favorable }\end{array}$ \\
\hline 11 & Hiperpolimenorrea & LH/FSH 9:1 & $\begin{array}{l}\text { HUA secundaria a } \\
\text { disfunción ovárica }\end{array}$ & $\begin{array}{l}\text { Medroxiprogesterona } \\
\text { Clormadinona }\end{array}$ & $\begin{array}{l}1 \\
1\end{array}$ & Falla al tratamiento \\
\hline
\end{tabular}

*Esta paciente tuvo dos evaluaciones.

AOC: anticonceptivos orales combinados; FSH: hormona folículo estimulante; HUA: hemorragia uterina anormal; LH: hormona luteinizante.

cuarta semana el sangrado se hizo más abundante y se cambió a medroxiprogesterona ( $10 \mathrm{mg} / \mathrm{día}$ ) durante siete meses.

Caso 6. Paciente valorada por polimenorrea, con descenso de $\mathrm{Hb}$ de $2 \mathrm{~g} / \mathrm{dl}$, que condiciona anemia, con $\mathrm{Hb}$ de $7 \mathrm{~g} / \mathrm{l}$. El ultrasonido reveló cistoadenoma de ovario. En un inicio se dio tratamiento con clormadinona a dosis $2 \mathrm{mg} /$ día durante nueve meses; sin embargo, tuvo falla al tratamiento (hiperpolimenorrea), por lo que se cambió a hormonales combinados una tableta/ día durante 21 días, obteniéndose buena respuesta.
Posteriormente, se realizó resección quirúrgica del cistoadenoma de ovario por persistir con irregularidades menstruales.

Caso 10. Paciente valorada por hiperpolimenorrea. Reultados de exámenes de laboratorio: $\mathrm{Hb}, 8.7 \mathrm{~g} / \mathrm{dl}$. El ultrasonido pélvico reveló ovarios poliquísticos. Se inició manejo con medroxiprogesterona (150 mg dosis única) para la inhibición de la menstruación, junto con manejo agudo de la hemorragia con clormadinona, y tuvo una respuesta favorable durante un mes; posteriormente, la paciente reinició con polimenorrea y se 
inició tratamiento con hormonales combinados con etinilestradiol/levonorgestrel $(0.03 \mathrm{mg} / 0.15 \mathrm{mg})$ con respuesta adecuada.

Caso 11. Paciente valorada por hiperpolimenorrea y anemia severa secundaria, con $\mathrm{Hb}$ de $3.6 \mathrm{~g} / \mathrm{dl}$, ameritando transfusión en dos ocasiones. Se decidió la supresión del sangrado menstrual en forma temporal por la anemia con medroxiprogesterona $150 \mathrm{mg}$ en una sola dosis, además de clormadinona para el manejo de la hemorragia, con respuesta parcial. En la actualidad, esta paciente se encuentra en vigilancia permanente.

\section{Pacientes con antecedentes de ciclos menstruales irregulares}

En las pacientes con antecedentes menstruales irregulares fue muy variable el tiempo de presentación de la hemorragia uterina anormal. Las pacientes 1, 4 y 7 tuvieron una respuesta favorable con el uso de progestágenos, mientras que el caso 5 tuvo una falla inicial por mal apego al tratamiento. El resto de las pacientes presentaron falla al tratamiento inicial. A continuación, se presentan estos casos.

Caso 9. Paciente valorada a los 13 años de edad por presentar hiperpolimenorrea y anemia leve $(\mathrm{Hb}$, $10.7 \mathrm{~g} / \mathrm{dl}$ ). Se detectó hirsutismo (escala Ferriman 8) y el ultrasonido reveló quiste de ovario derecho. Se inició tratamiento con hormonales combinados con etinilestradiol/desogestrel a razón de dos tabletas cada 24 horas, cediendo el sangrado a las 48 horas. Posteriormente se indicó una tableta cada 24 horas hasta completar 21 días, esquema que continuó durante cuatro meses con buena respuesta. Al cuarto mes de tratamiento un médico no especialista decidió cambiar a clormadinona $2 \mathrm{mg} / \mathrm{día}$ en la segunda fase del ciclo menstrual; sin embargo, presentó nuevamente hiperpolimenorrea, por lo que se inhibió la menstruación con medroxiprogesterona (150 mg cada 21 días) durante seis meses. Al séptimo mes, la paciente presentó nuevamente sangrado transvaginal abundante, por lo que, en conjunto con el servicio de Endocrinología, recibió manejo análogo de GnRh (aGnRH) a razón de $3.75 \mathrm{mg} /$ mes, con buena respuesta durante 12 meses. Después de la suspensión de los aGnRH y hasta aproximadamente 9 meses se mantuvo sin problemas; posteriormente presentó el mismo tipo de alteraciones menstruales. Se hizo una nueva valoración integral, y se documentó un quiste simple en el ovario derecho; recibió manejo con clormadinona $2 \mathrm{mg} /$ día durante dos meses con buena respuesta clínica. Posteriormente se cambió a hormonales orales combinados con etinilestradiol/levonorgestrel $(0.03 \mathrm{mg} / 0.15 \mathrm{mg})$ una tableta/día, continuando con ciclos regulares durante otros cuatro meses. Se dio de alta por mayoría de edad dando indicaciones de continuar con los hormonales combinados.

\section{Discusión}

Las alteraciones menstruales en adolescentes son muy comunes, en particular en los primeros años posteriores a la menarca, en vista, fundamentalmente, de la falta de maduración del eje hipotálamo-hipófisis-ovario. La evaluación y tratamiento de las adolescentes con enfermedades crónicas, quienes además del problema de base presentan trastornos menstruales, son motivo de preocupación para el equipo de salud. En especial, en el caso de las pacientes con ERC, cuando se presentan con menstruaciones abundantes, puede ser complicado mejorar los niveles de hemoglobina ${ }^{5,9}$.

Desde hace años existen diferentes recomendaciones y guías para el manejo de los trastornos menstruales en adolescentes. En estos documentos se pone de manifiesto que el tratamiento debe basarse en las condiciones generales de cada paciente (estado hemodinámico y niveles de hemoglobina), así como en la cantidad del sangrado menstrual. En general, en casos de sangrado agudo y abundante, la mejor conducta sería la inhibición de la menstruación, y cuando el sangrado menstrual es prolongado e irregular pero no tan profuso, entonces el objetivo del tratamiento se enfocaría a la regulación de los ciclos menstruales ${ }^{11-14}$.

A pesar de estas recomendaciones, es muy limitado el número de publicaciones sobre la experiencia del tratamiento de los trastornos menstruales en pacientes con enfermedades crónicas, y las pacientes con ERC no son la excepción. En este último grupo de pacientes fue muy interesante descubrir, después de realizar una búsqueda amplia de la bibliografía sobre el tema, que no hay estudios donde se haya documentado la experiencia de lo que ocurre en otros centros hospitalarios. Las diferentes publicaciones que pudimos localizar se refieren al reporte de casos aislados ${ }^{15-18}$.

En cuanto al proceso diagnóstico, también es necesario destacar que en todas las pacientes se lleva a cabo un escrutinio de cada uno de los aspectos que se recomiendan ante un caso de trastorno menstrual12,20-23. Así, para cada paciente deben solicitarse niveles de hormonas y ultrasonido pélvico, con el propósito de identificar posibles causas y de orientar el tipo de tratamiento. De ahí que, como se observó en 
los resultados mostrados, se pudieron identificar trastornos como hipotiroidismo, cistoadenoma de ovario y presencia de quiste simple de ovario.

Es importante destacar que hubo dos pacientes en quienes fue necesaria la atención unos meses después de presentar la menarca. Si bien se espera la presentación de alteraciones menstruales por la inmadurez del eje hipotálamo-hipófisis-gónada, el riesgo de descompensación hemodinámica en estas pacientes fue alto y no permitía mantener un manejo expectante.

Con respecto al tratamiento, en términos generales permitió controlar el sangrado menstrual en la mayoría de las pacientes. Como se describió, en casi todos los casos, el primer esquema de tratamiento se basó en la disminución o inhibición del sangrado con progestágenos (más frecuentemente clormadinona) y, posteriormente, el uso de anticonceptivos combinados para la regularización de los ciclos ${ }^{13,14,24,25}$. Sin embargo, también se pudo observar que existen pacientes en las que, a pesar de seguir el mismo esquema de tratamiento, no fue posible controlar el sangrado de una manera óptima, por lo que fue necesario el uso de clormadinona y medroxiprogesterona por tiempo prolongado. Al respecto, en los pocos casos de la literatura se describen pacientes que, ante la falta de respuesta a estos fármacos, han requerido el uso de otras intervenciones, como la ablación endometrial o la utilización del dispositivo intrauterino (DIU) con levonorgestrel15,16. Otro aspecto importante a considerar en estas pacientes son los efectos colaterales que tienen los hormonales utilizados, tales como los estrógenos, que incrementan el riesgo de trombosis, y los progestágenos, por el riesgo de condicionar descontrol hipertensivo por retención hídrica, lo que contribuye al manejo complejo de las pacientes con ERC. Estos aspectos deben ser estrechamente vigilados, principalmente en pacientes que se encuentran en hemodiálisis, y sobre todo con fístulas arteriovenosas.

A pesar que este es el primer estudio donde se informa de manera más amplia del pronóstico de este tipo de pacientes, y en el que los hallazgos son alentadores debido a la buena respuesta en general que presentaron las adolescentes con ERC y trastornos menstruales, es importante reconocer las limitaciones de este trabajo. La principal es que se trata de un estudio retrospectivo donde toda la información se obtuvo del expediente clínico. Esta situación no permitió encontrar toda la información necesaria para la mejor descripción de cada uno de los casos. Por ejemplo, a pesar que la evaluación del estadio puberal y el análisis del calendario menstrual es una actividad que siempre se lleva a cabo durante la consulta de Ginecología, no en todas las notas clínicas estaba descrito; lo mismo sucedió con los datos puntuales de las diferentes determinaciones de los niveles hormonales. Además, se pudo comprobar que no existe un criterio uniforme y objetivo para evaluar el comportamiento de las pacientes una vez que se inicia el tratamiento encaminado al control del sangrado menstrual. En este estudio, para la descripción de la evolución de cada paciente, fue necesario revisar con detalle cada nota clínica para definir si la terapia había sido o no efectiva o favorable.

Otra limitante fue no haber considerado como una posible variable de confusión la uremia, independientemente del tipo de terapia de sustitución, ya que se ha observado que cuanto mejor sea la dialisancia, los trastornos menstruales son menores. Tampoco se contempló el estado de nutrición, y tanto la desnutrición como la obesidad son causas de trastornos menstruales ${ }^{25}$. Sin embargo, se debe tener en cuenta que este es un estudio retrospectivo, por lo que sería muy difícil establecer de manera confiable el estado de nutrición de los pacientes en ERC en los diferentes momentos en que se presentaron los trastornos menstruales.

Por lo anterior y en vista de los resultados obtenidos en el presente trabajo, se espera continuar con la forma en que se realiza el proceso diagnóstico-terapéutico de este grupo de pacientes, registrando y documentando de manera más objetiva cada uno de los casos que en el futuro se presenten, a fin de disponer de mejor información. Otro punto primordial será tratar de que se incluya, como parte de la evaluación integral en todas las adolescentes con ERC, el escrutinio de su patrón menstrual. Además, será interesante buscar intencionadamente a las adolescentes con ERC que tienen amenorrea u oligomenorrea, ya que es un grupo que no está representado en este trabajo.

En conclusión, las adolescentes con ERC con hemorragia uterina anormal requieren de una evaluación integral que incluye la determinación de niveles séricos de hormonas, así como la realización de ultrasonido pélvico. Basándonos en la experiencia de la Clínica de Ginecología Pediátrica, el tratamiento con progestágenos 0 anticonceptivos orales demostró ser efectivo.

\section{Responsabilidades éticas}

Protección de personas y animales. Los autores declaran que para esta investigación no se han realizado experimentos en seres humanos ni en animales. 
Confidencialidad de los datos. Los autores declaran que han seguido los protocolos de su centro de trabajo sobre la publicación de datos de pacientes.

Derecho a la privacidad y consentimiento informado. Los autores han obtenido el consentimiento informado de los pacientes y/o sujetos referidos en el artículo. Este documento obra en poder del autor de correspondencia.

\section{Bibliografía}

1. Kerkhoff BA, O'Connor TC, Plant WD, Higgins Jr. Poor uptake of reproductive health screening services by female renal transplant recipients. Ir Med J. 2006;99:78-80

2. Lane PH. Puberty and chronic kidney disease. Adv Chronic Kidney Dis. 2005;12:372-7.

3. Karagiannis A, Harsoulis F. Gonadal dysfunction in systemic diseases. Eur J Endocrinol. 2005;152:501-13.

4. Hawkins E, Chudnoff S, Levie M, Fridman D, Mehta S, Melamed M. Menstrual abnormalities and reproductive challenges in women with end stage renal disease on chronic dialysis. J Minim Invasive Gynecol. 2015;22:S74-5.

5. Biff FP. Endocrine Disorders in Renal Failure. En: Singh A, Williams G. Textbook of nephro-endocrinology. San Diego, CA: Elsevier; 2009. pp 437-9.

6. Holley JL. The hypothalamic-pituitary axis in men and women with chronic kidney disease. Adv Chronic Kidney Dis. 2004;11:331-7.

7. Chakhtoura Z, Meunier M, Caby J, Mercadal L, Arzouk N, Barrou B, et al Gynecologic follow up of 129 women on dialysis and after kidney transplantation: a retrospective cohort study. Eur J Obstet Gynecol Reprod Biol. 2015;187:1-5.

8. Atallah D, Salameh C, El Kassis N, Safi J, Lutfallah F, Bejjani L, et al. Infertility and kidney transplantation. J Med Liban. 2015;63:138-43.

9. Ghazizadeh S, Lessan-Pezeshki M. Reproduction in women with end-stage renal disease and effect of kidney transplantation. Iran J Kidney Dis. 2007;1:12-5.
10. Wolf G, Chen S, Han D, Ziyadeh F. Leptin and renal disease. Am J Kidney Dis. 2002;39:1-11.

11. Jamieson MA. Disorders of menstruation in adolescent girls. Pediatr Clin North Am. 2015;62:943-61.

12. Slap GB. Menstrual disorders in adolescence. Best Pract Res Clin Obstet Gynaecol. 2003;17:75-92.

13. Hayden G, Emans J. Abnormal vaginal bleeding in adolescents. Pediatr Rev. 2007;28:175-82.

14. Fedele L, Gammaro L, Bianchi S. Levonorgestrel-releasing intrauterine device for the treatment of menometrorrhagia in a woman on hemodialysis. N Engl J Med. 1999;341:541.

15. Zurawin RK, Pramanik S. Endometrial balloon ablation as a therapy for intractable uterine bleeding in an adolescent. J Pediatr Adolesc Gynecol. 2001:14:119-21.

16. Hedges SJ, Dehoney SB, Hooper JS, Amanzadeh J, Busti AJ. Evidence-based treatment recommendations for uremic bleeding. Nat Clin Pract Nephrol. 2007;3:138-53.

17. Filocamo MT, Zanazzi M, Li Marzi V, Lombardi G, Del Popolo G, Mancini $G$, et al. Sexual dysfunction in women during dialysis and after renal transplantation. J Sex Med. 2009;6:3125-31.

18. Levey A, Eckardt K, Tsukamoto Y, Levin A, Coresh J, Rossert J, et al. Definition and classification of chronic kidney disease: a position statement from Kidney Disease: Improving Global outcomes (KDIGO). Kidney Int. 2005;67:2089-100

19. Gayon E, Sam S, Soto S. Hemorragia genital anormal en niñas y adolescentes. Abordaje clínico y terapéutico. Acta Pediatr Mex. 2006;27:84-95.

20. Delitala A, Fanciulli G, Maioli M, Delitala G. Subclinical hypothyroidism, lipid metabolism and cardiovascular disease. Eur J Intern Med. 2017:38:17-24.

21. Halperin Rabinovich I, Cámara Gómez R, García Mouriz M, Ollero García-Agulló D; Grupo de Trabajo de Neuroendocrinología de la SEEN. Clinical guidelines for diagnosis and treatment of prolactinoma and hyperprolactinemia. Endocrinol Nutr. 2013;60:308-19.

22. Muxí-Moner $C$, Jordà-García B. Abordaje práctico de las metrorragias. FMC. 2009;16:475-9.

23. Templeman $C$, Hertweck S. Vaginal Bleeding in Childhood and Menstrual Disorders in Adolencence. En: Sanfilipo JS, Muran D. editores. Pediatric and adolescent gynecology. Philadelphia: Saunders; 2001. pp 237-47.

24. Rager KM, Fowler A, Omar HA. Successful treatment of depot medroxyprogesterone acetate-related vaginal bleeding improves continuation rates in adolescents. ScientificWorldJournal. 2006;6:353-5.

25. Serret-Montoya J, Hernández-Cabezza A, Mendoza O, Cárdenas NR Villasis KM. Alteraciones menstruales en adolescentes. Bol Med Hosp Infant Mex. 2012;69:63-76. 